\title{
The smart era of electrochemical energy storage devices
}

\author{
Xu-Yi Shan ${ }^{1}$, Feng Li ${ }^{1, *}$, Da-Wei Wang ${ }^{2}$ and Hui-Ming Cheng ${ }^{1}$
}

1. Shenyang National Laboratory for Materials Science, Institute of Metal Research, Chinese Academy of Sciences, Shenyang, 110016, China

2. School of Chemical Engineering, The University of New South Wales, Sydney, NSW 2052, Australia

*E-mail: fli@imr.ac.cn

Smart devices were proposed by Mark Weiser in 1991, and are electronic devices that can, to some extent, operate interactively and autonomously [1]. During the following two decades, the world of intelligent technology has grown fast and has greatly changed communication, transportation and our daily lives.

As we all know, the rapid development of portable electronic devices, electric vehicles, and large-scale grid energy storage has demanded a large number of electrochemical energy storage devices (EESDs) with higher energy density, longer cycle life, higher reliability, and especially, better safety [2-6]. However, an EESD is a complicated system with many components (cathode, anode, separator, electrolyte, and packaging) involving various electrochemical mechanisms. Although voltage and current changes are apparent after EESD assembly and during use, very little or nothing is known about the changes that occur in the internal structure of individual components during electrochemical charge/discharge, which are strongly related to device safety. Hence, with the global use of EESDs, safety issues such as thermal runaway, overcharging, dendrite formation, gassing and electrolyte decomposition can unpredictably and randomly emerge during the use of electric appliances. These can lead to explosions, fires and even casualties if left uncontrolled. To minimize these failures, a complete awareness of the environment inside EESDs and prompt feedback is necessary but challenging to achieve.

At the same time, the massive use of EESDs brings another big challenge---how to deal with the degraded EESDs? The conventional method is recycling, which can 
lessen the environmental impact of batteries, as well as the environmental harm caused by the disposal of hazardous battery materials [7]. However, today's methods of commercial recycling involve nothing more than the extraction of metals from the cathode, which is based on metal refining. The first and most widespread method is pyrometallurgy, in which EESDs are fed into a smelter and melted down to recover the metals. However, the process is complex, energy consuming and uneconomic [7]. How to recycle the non-metal components of the EESDs and minimize their environmental harm remains a big challenge. For practical appliances, particularly portable electronic products, embedded EESDs are the mainstream choice for power supplies [8]. The life of these embedded EESDs determines the service life of these electronics. Hence, finding ways to regenerate the degraded EESDs instead of disposal or recycling would be a big advance but is also very challenging.

How can one overcome these challenges? The best answer may be by using smart EESDs. Smart control of EESDs can provide us with more information about their use and allow them to work automatically and interactively with electric appliances. The commercial smart part of a battery pack is a built-in battery management system (BMS). Benefitting from sensors and feedback systems inside a BMS, we can measure voltage and current changes, deduce the charge level, manage heat distribution, balance capacity and communicate with electric appliances. For example, good design of the BMS in a Tesla model S P85D automobile ensures the accurate cooperation of more than seven thousand 18650 form-factor Li-ion cells to deliver ultrafast acceleration (0 100 km/h in 3 seconds) safely [9]. Recently, Microsoft Corporation has proposed a software-defined battery (SDB) system to smartly manage different battery types inside a battery pack to meet the wide range of user requirements [10]. Through interactions between the batteries, their chemistry, the battery management circuit and the operating system (OS), the SDB allows the device to use different batteries by fine tuning the amount of charge flowing in and out of each battery by a " $\mu$ controller". The SDB is a big leap forward for smart EESDs at the pack-level.

Although significant improvement of user experience with EESDs has been 
achieved by using a BMS or SDB at the pack-level, the potential hazards inside a cell are still disconcerting, such as electrode working conditions, status of the electrolyte, lithium dendrite formation, etc. Therefore, smart design at the cell-level is urgently needed from a system design viewpoint.

The first smart cell design that allowed the early detection of lithium dendrite growth inside a battery was reported by $\mathrm{Wu}$ et. al. [11]. The configuration allows internal lithium dendrite growth to be monitored in-situ by using a polymer-copperpolymer triple layer separator (Fig. 1). The copper was deposited by magneto-sputtering and did not alter the ion conductivity of the separator. For conventional lithium batteries, the lithium dendrite is only detected when the battery fails due to an internal short circuit, as shown in Fig. 1a. However, in the smart cell configuration (Fig. 1b) the copper layer is connected to a third terminal, and once a dendrite grows and connects with the copper layer, a sharp voltage drop between the copper layer and the anode from $3 \mathrm{~V}$ to almost zero is immediately detected (Fig. 1c). At the same time, the cell is not internally short-circuited and remains safely operational with a non-zero potential (Fig. 1d). On detection of this distinctive signal, the cell can be removed from service, effectively preventing fire and explosion. 
(a)
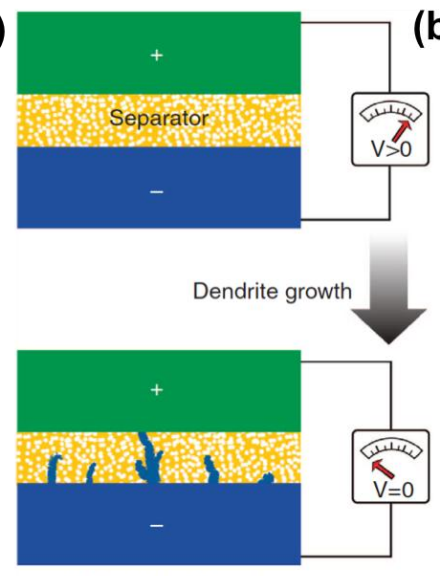

Internal short-circuit (possible explosion hazard) (b)
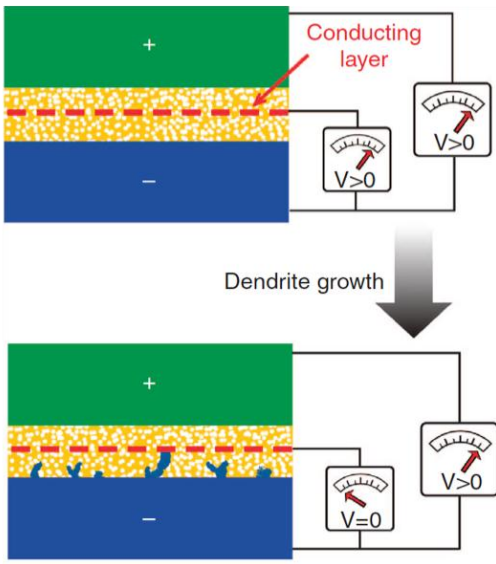

Dendrite detected (battery still safe)

(c)

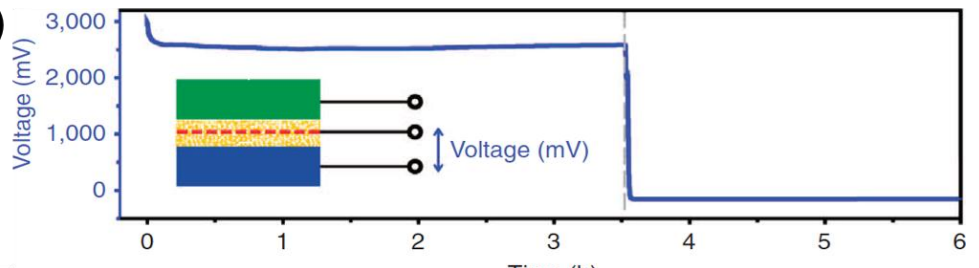

(d)

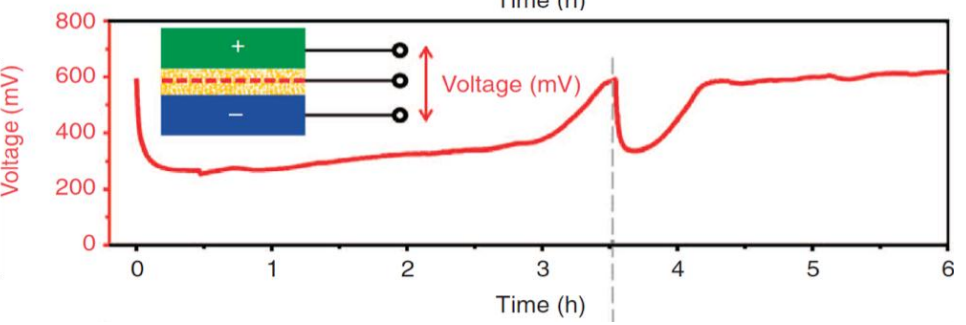

Figure 1. Schematic and experimental demonstration of the smart cell design. (a) Dendrite formation in a conventional lithium battery. (b) The smart cell with a bi-functional separator gives rise to a warning of impending failure due to an internal short circuit. (c) Voltage profile of a Li/Li battery with a bifunctional separator, where

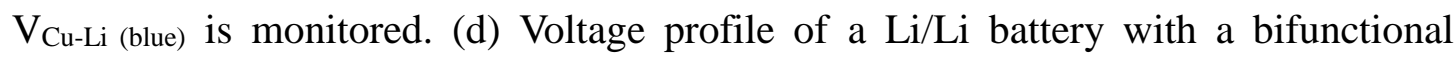
separator, where $\mathrm{V}_{\mathrm{Li}-\mathrm{Li} \text { (red) }}$ is monitored. [11]. Copyright 2014, Nature Publishing Group.

Very recently, our group has developed a smart lithium ion supercapacitor (SLIC), as shown in Fig. 2. Compared to the conventional EESDs (Fig. 2a), a SLIC has an additional lithium electrode as a voltage modulator and two voltage sensors to provide feedback (Fig. 2b) [12]. As a result of the voltage modulator and the feedback, the proof-of-concept SLIC has three smart functions:

(1) Energy boosting. An electrochemical charge injection (ECI) protocol was 
developed to maximize the energy density of the supercapacitors [13]. Here, the voltage modulator serves as a lithium source for the ECI (Fig. 2c) and boost energy (Fig. 2d).

(2) Safety monitoring. Changes in the potential of each electrode can be monitored in real-time by the voltage sensors $\left(\mathrm{V}_{1}\right.$ and $\left.\mathrm{V}_{2}\right)$ in the feedback (Fig. 2c). During cycling, when $\mathrm{V}_{2}>4.5 \mathrm{~V}$ (electrolyte decomposition) or $\mathrm{V}_{1}<0 \mathrm{~V}$ (lithium plating), the sensors send an alert and the device is turned off (Fig. 2e). This effectively prevents swelling and Li dendrite formation, which may cause fire and explosion if left uncontrolled.

(3) Self-regeneration. In a conventional system, the degraded SLIC that was turned off by the feedback will be discarded or recycled, but here another ECI operation (Fig. 2c) will regenerate it. Following a $40 \%$ decay (red curve in Fig. 2f), the capacity of the SLIC is fully recovered by self-regeneration (blue curve in Fig. 2f). Instead of disposal, the self-regeneration offers an environmentally-friendly way of dealing with the degraded cell.

(a) Conventional EESDs:
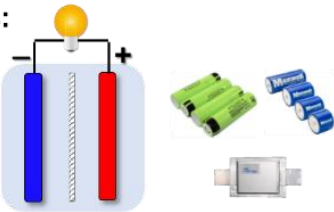

(b) SLIC:
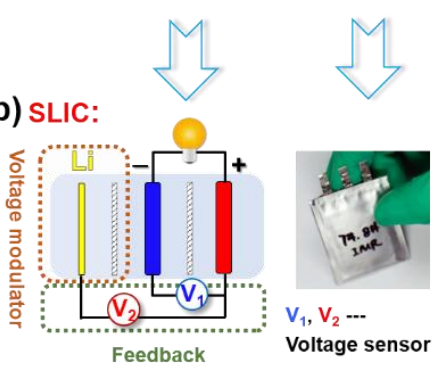

(c) SLIC $\rightarrow$ Feedback + Voltage modulator

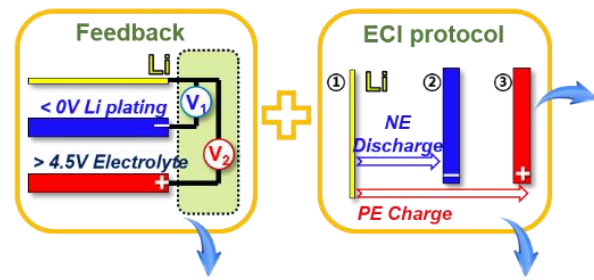

(e) (2) Safety monitoring

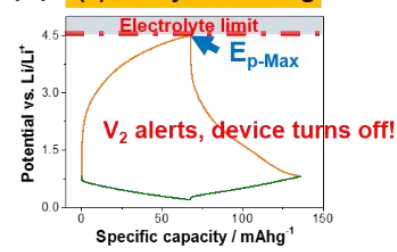

(d) (1) Energy boosting

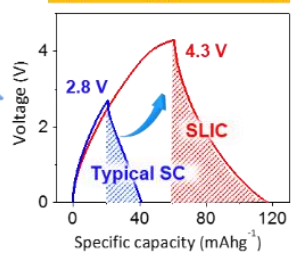

(f) (3) Self-regeneration

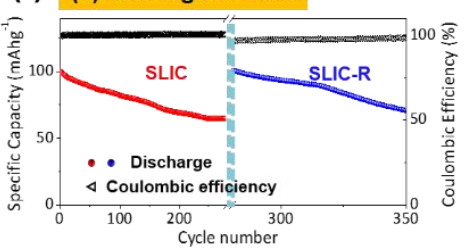

Figure 2. Schematic of the configuration and smart functions of a SLIC. Compared to a conventional EESD with two electrodes, a SLIC has an additional lithium voltage modulator and two voltage sensors. The proof-of-concept SLIC has three smart functions: (1) energy boosting; (2) safety monitoring; (3) self-regeneration [12]. Copyright 2015, Elsevier. 
In summary, smart EESDs have shown great potential for improving user experience. Beside the development of a BMS, a smart cell design at the cell-level is equally important. As shown in Fig. 3, future cooperation of a smart cell design at the cell-level and the development of a BMS at the battery/pack-level will strongly increase the interaction between EESDs and users. Smart EESDs can greatly improve the user experience, such as private customization, on-demand supply, fast charging, and safety control. They can also improve user experience by adjusting their own status based on the users' behavior. Furthermore, data analysis of the users' behavior by the internal sensors and the related software APPs will provide positive feedback to optimize the design. Above all, smart EESDs can work as a bridge that connects users and $R \& D$ design engineers to create a safer and more intelligent electrochemical energy storage future.

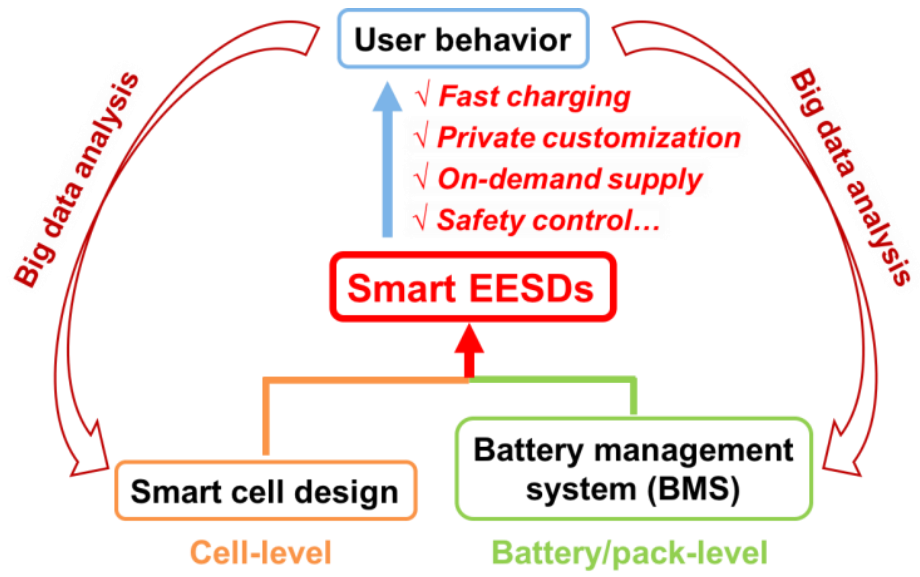

Figure 3. Perspective for smart EESDs.

\section{Acknowledgement}

This work is supported by Ministry of Science and Technology of China (No. 2014CB932402), National Science Foundation of China (Nos. 51221264, 51525206, 51172239, 51372253 and U1401243), "Strategic Priority Research Program" of the Chinese Academy of Sciences (XDA01020304), the Key Research Program of the Chinese Academy of Sciences (Grant No. KGZD-EW-T06), and Research Supported by the CAS/SAFEA International Partnership Program for Creative Research Teams. 


\section{REFERENCES}

[1] M. Weiser, Sci. Am., 1991, 265, 94.

[2] P. Simon and Y. Gogotsi, Nat. Mater., 2008, 7, 845.

[3] J. Yan, Q. Wang, T. Wei and Z. J. Fan, Adv. Energy Mater., 2014, 4, 1300816.

[4] J. M. Tarascon, M. Armand, Nature, 2001, 414, 359.

[5] N.-S. Choi, Z. Chen, S. A. Freunberger, X. Ji, Y.-K. Sun, K. Amine, G. Yushin, L. F. Nazar, J. Cho and P. G. Bruce, Angew. Chem., 2012, 51, 9994.

[6] C. Liu, F. Li, L. P. Ma and H. -M. Cheng, Adv. Mater., 2010, 22, E28.

[7] E. Gies, Nature, 2015, 526, S100.

[8] G. J. Jeong, Y. -U. Kim, H. S. Kim., Y. -J. Kim and H. -J. Sohn, Energy Environ. Sci., 2011, 4, 1986.

[9] Tesla Model S. https://www.teslamotors.com.

[10] A. Badam, R. Chandra, J. Dutra, A. Ferrese, S. Hodges, P. Hu, J. Meinershagen, T. Moscibroda, B. Priyantha and E. Skiani, Proceedings of the 25th Symposium on Operating Systems Principles, 2015, 215.

[11] H. Wu, D. Zhuo, D. S. Kong and Y. Cui, Nat. Comm., 2014, 5, 5193.

[12] X. -Y. Shan, Y. Z. Wang, D. -W. Wang, Z. Weng, F. Li and H. -M. Cheng, Energy Stor. Mater., 2015, 1, 146.

[13] Z. Weng, F. Li, D. -W. Wang, L. Wen and H. -M. Cheng, Angew. Chem., 2013, 52, 3722. 